DFTT 64/93

October 1993

\title{
PERTURBATIVE QCD FORBIDDEN CHARMONIUM DECAYS AND GLUONIA
}

\author{
M. Anselmino ${ }^{a}$, M. Genovese $^{a}$ and D.E. Kharzeev ${ }^{b, c}$ \\ ${ }^{a}$ Dipartimento di Fisica Teorica, Università di Torino \\ and INFN, Sezione di Torino, 10125 Torino, Italy \\ ${ }^{b}$ INFN, Sezione di Pavia, 27100 Pavia, Italy \\ ${ }^{c}$ Moscow University, 119899, Moscow, Russia
}

\begin{abstract}
We address the problem of observed charmonium decays which should be forbidden in perturbative QCD. We examine the model in which these decays proceed through a gluonic component of the $J / \Psi$ and the $\eta_{c}$, arising from a mixing of $(c \bar{c})$ and glueball states. We give some bounds on the values of the mixing angles and propose the study of the $p \bar{p} \rightarrow \phi \phi$ reaction, at $\sqrt{s} \simeq 3 \mathrm{GeV}$, as an independent test of the model.
\end{abstract}




\section{Introduction}

It has been known for many years that the $J / \Psi$ and the $\eta_{c}$ decay respectively into Vector-Pseudoscalar (VP) and Vector-Vector (VV) mesons, channels which should be forbidden by the helicity conservation rule in perturbative QCD and the usual assumption of the dominance of collinear valence quark configurations; a similar problem holds for the decay of $\eta_{c}$ into $p \bar{p}$ 1, 2, 3, 4,

Several attempts to overcome such difficulties by taking into account and modeling non perturbative effects can be found in the literature [3, 5, 6]. Two quark correlations inside the proton, or diquarks, although very likely present in the $Q^{2}$ region of charmonium decays, do not seem to help with the $\eta_{c} \rightarrow p \bar{p}$ large observed decay rate [5]; quark mass corrections are equally of little help with $\eta_{c} \rightarrow p \bar{p}$ [6] and do not contribute at all to $\eta_{c} \rightarrow V V$ decays [3].

Apart from eventual effects due to the intrinsic transverse motion of quarks inside the hadrons, which have not yet been investigated, at this stage two possible phenomenological explanations of the above problems remain: one requires a large contribution due to twist three operators or higher order Fock components of the hadron wave functions [2], and the second assumes a mixing of the $J / \Psi$, [4, 7] and of the $\eta_{c}$ [8] with a glueball. We shall argue that, at the light of recent experimental data, the latter assumption seems to us a more realistic explanation of the puzzle; it certainly is a more interesting one, in that it amounts to allow for the presence of the fundamental trigluonium states, which have the quantum numbers $J^{P C}=$ $0^{-+}, 1^{--}, 3^{--}$, around a mass of $3 \mathrm{GeV} / c^{2}$ [8], in agreement with the prediction of some models 9 .

In the next Sections, after a brief discussion of the higher twist model and its comparison with data, we shall further investigate the glueball mixing idea giving a first rough estimate of the values of the mixing angles and proposing some experimental tests which could shed some light on the subject in the near future.

\section{$2 \quad$ Higher Twist Model}

In Ref. [2] it has been suggested, in a consistent perturbative QCD treatment, that the $J / \Psi$ decays into $V P$ could be explained through the introduction of twist three meson wave functions, namely through the $\mid q \bar{q} g>$ Fock components of the $V$ and $P$ mesons. Detailed calculations, in good agreement with the data, have been performed for the branching ratio $B(J / \Psi \rightarrow \rho \pi)$. Similarly, the double OZI suppressed decay $\eta_{c} \rightarrow \omega \phi$ has been computed and the result exploited, using $S U(3)$ symmetry and an effective lagrangian, to estimate some relative magnitudes of other $\eta_{c} \rightarrow V V$ branching ratios [10].

The first conceptual difficulty of this model is given by the necessity of an unexpectedly large contribution from a higher Fock component, also considering the 
theoretical problems connected with the study of three-particle wave functions [1]. The main problem, however, is the fact that such scheme should predict analogous results for the $\Psi^{\prime}$ decays, whereas the decays $\Psi^{\prime} \rightarrow P V$ are observed to be strongly suppressed: $\operatorname{Br}\left(\Psi^{\prime} \rightarrow \rho \pi\right)<8.3 \cdot 10^{-5}, \operatorname{Br}\left(\Psi^{\prime} \rightarrow K \bar{K}^{*}\right)<1.79 \cdot 10^{-5}$ [12].

Furthermore, some other predictions of the model are not in agreement with the experimental data:

i) in Ref. [10] the branching ratios of $\eta_{c}, \chi_{0}$ and $\chi_{2}$ in the double OZI suppressed channel $\omega \phi$ are predicted to be roughly of the same order of magnitude as the single OZI suppressed ones. However, such double OZI suppressed decays have not yet been observed, whereas the single OZI suppressed decays into VV have been clearly observed.

ii) Ref. [10 predicts

$$
\frac{\operatorname{Br}\left(\eta_{c} \rightarrow \omega \omega\right)}{\operatorname{Br}\left(\eta_{c} \rightarrow \phi \phi\right)} \simeq 1.3
$$

in disagreement with the experimental information

$$
\frac{B r\left(\eta_{c} \rightarrow \omega \omega\right)}{B r\left(\eta_{c} \rightarrow \phi \phi\right)}<0.44 .
$$

Even inserting into Eqs. (38)-(39) of Ref. [10] updated experimental data, one obtains for Eq. (11) the value $\simeq 0.8$, still in disagreement with Eq. (2).

iii) finally, no direct computation of $\eta_{c} \rightarrow V V(V, V \neq \omega, \phi)$ with three-particle wave functions has been attempted, and no attempt at all has been made to compute the $\eta_{c} \rightarrow p \bar{p}$ decay rate.

We must accept that higher twist contributions still leave many unsolved problems; we then turn to a different possible solution and from now on we concentrate on the glueball mixing model.

\section{Mixing Parameters}

We adopt the following mixing schemes:

$$
\begin{gathered}
|J / \Psi>=\cos \theta| c \bar{c}>_{J / \Psi}+\sin \theta \mid O> \\
\left|\eta_{c}>=\cos \alpha\right| c \bar{c}>_{\eta_{c}}+\sin \alpha \mid P>
\end{gathered}
$$

where $O$ is a vector and $P$ a pseudoscalar glueball.

Due to perturbative QCD helicity conservation it follows:

$$
\begin{aligned}
& <V P \mid c \bar{c}>_{J / \Psi}=0 \\
& <V P|J / \Psi>=\sin \theta<V P| O>
\end{aligned}
$$

and due to the (lowest order) decoupling of gluons from photons, 


$$
\begin{aligned}
<e^{+} e^{-} \mid O> & =0 \\
<e^{+} e^{-} \mid J / \Psi> & =\cos \theta<e^{+} e^{-} \mid c \bar{c}>_{J / \Psi} .
\end{aligned}
$$

Analogous equations are valid, mutatis mutandis, for the pseudoscalar case $(O \rightarrow$ $\left.P, J / \Psi \rightarrow \eta_{c}, V P \rightarrow V V, e^{+} e^{-} \rightarrow \gamma \gamma\right)$

In order to obtain an, admittedly rough, estimate of the mixing angles we need at least an estimate of the total width of the $O(P)$ and of the partial width of the $O(P)$ into Vector-Pseudoscalar (Vector-Vector) mesons. A possible way to get the former is offered by the $\sqrt{O Z I}$ suppression of the glueball decay rule [13], which suggests the width of a trigluonium to be in between the one for a light quark state with a mass around $3 \mathrm{GeV} / c^{2}$ and the $J / \Psi$ one, namely

$$
\Gamma_{O} \approx \sqrt{\Gamma_{J / \Psi} \cdot 500 \mathrm{MeV}} \approx 7 \mathrm{MeV}
$$

and the width of a digluonium to be beteewn the one for a light quark state with a mass around $3 \mathrm{GeV} / c^{2}$ and the $\eta_{c}$ one, namely

$$
\Gamma \approx \sqrt{\Gamma_{\eta_{c}} \cdot 500 \mathrm{MeV}} \approx 70 \mathrm{MeV}
$$

Such a rule, however, has been criticized on various grounds [14], and in the following we will assume the values in Eq. (9) and (10) as lower bounds, taking

$$
\Gamma_{O} \approx \Gamma_{P} \approx(10 \div 100) \mathrm{MeV}
$$

This estimate is consistent with the value suggested in Ref. [7].

In order to estimate $\Gamma_{O \rightarrow V P}$ we consider that a $1^{--}$state is allowed to decay into eight different couples of meson nonets: $V P, S V, V T, P A^{+}, S A^{-}, V A^{+}, T A^{-}$, $A^{+} A^{-}$; therefore, if we consider a full flavour democracy in the glueball's decays and ignore differences in phase spaces, we have that (approximating to $1 / 10$ in order to include also eventual excited nonets and three body decays)

$$
\Gamma_{O \rightarrow V P} \approx \frac{1}{10} \Gamma_{O} \approx(1 \div 10) \mathrm{MeV}
$$

Analogously, for the pseudoscalar state we have 13 channels: $P P, P S, P T, S S$, $S T, V V, T T, P A^{-}, S A^{+}, V A^{-}, T A^{+}, A^{+} A^{+}, A^{-} A^{-}$, and also in this case, within our approximations, we can assume

$$
\Gamma_{P \rightarrow V V} \approx \frac{1}{10} \Gamma_{P} \approx(1 \div 10) \mathrm{MeV}
$$

Of course $\Gamma_{O \rightarrow \rho \pi}\left(\Gamma_{P \rightarrow \rho \rho}\right)$ is a fraction of the above width; however, considered that the decays into VP (VV) are favoured with respect to the decays into the other couples of nonets by phase space, we can assume as a rough estimate:

$$
\Gamma_{O \rightarrow \rho \pi} \lesssim(1 \div 10) \mathrm{MeV}
$$




$$
\Gamma_{P \rightarrow \rho \rho} \lesssim(1 \div 10) \mathrm{MeV} .
$$

Using Eq. (14) we obtain from Eqs. (3) and (6)

$$
\Gamma_{J / \Psi \rightarrow \rho \pi}=1.09 \mathrm{keV} \approx \sin ^{2} \theta \cdot \Gamma_{O \rightarrow \rho \pi} \lesssim(1 \div 10) \cdot \sin ^{2} \theta \mathrm{MeV}
$$

which leads to

$$
\sin ^{2} \theta \gtrsim\left(10^{-4} \div 10^{-3}\right)
$$

On the other hand we must have

$$
\Gamma_{J / \Psi}>\sin ^{2} \theta \Gamma_{O}
$$

which gives

$$
\sin ^{2} \theta<8.6 \cdot\left(10^{-4} \div 10^{-3}\right)
$$

Analogously we have for the pseudoscalar case, from Eqs. (15) and (49):

$$
\Gamma_{\eta_{c} \rightarrow \rho \rho}=0.267 \mathrm{MeV} \approx \sin ^{2} \alpha \cdot \Gamma_{P \rightarrow \rho \rho} \lesssim(1 \div 10) \cdot \sin ^{2} \alpha \mathrm{MeV}
$$

which leads to

$$
\sin ^{2} \alpha \gtrsim 2.7 \cdot\left(10^{-2} \div 10^{-1}\right)
$$

and

$$
\Gamma_{\eta_{c}}>\sin ^{2} \alpha \Gamma_{P}
$$

which gives only the trivial bound

$$
\sin ^{2} \alpha<(0.1 \div 1)
$$

\section{Tests of the model}

The above results clearly show how a tiny admixture of glueballs in $\eta_{c}$ and $J / \Psi$ could be sufficient to explain the otherwise problematic charmonium decays. Let us then consider other consequences and predictions of the mixing scheme.

It is clear that, away from the glueball mass region, the model under investigation cannot help any more and perturbative QCD predictions should be valid; as a consequence, one expects the decays $\eta_{c}^{\prime} \rightarrow V V, p \bar{p}[8]$ and $\Psi^{\prime} \rightarrow V P$ [7] to be quite suppressed, as the mass difference between these states and the glueballs is much bigger and therefore the eventual mixing much reduced. Indeed the branching ratios for the decays $\Psi^{\prime} \rightarrow V P$ are known to be very small [12] and no $\eta_{c}^{\prime}$ has been observed up to now in $p \bar{p}$ annihilation [15].

Of course, also the decays $\eta_{b} \rightarrow V V, p \bar{p}$ and $\Upsilon \rightarrow V P$ should not be allowed, in agreement with perturbative QCD, there being no reason to have a glueball in the large mass range of these states. 
A direct observation of the glueball state in the proximity of the $\eta_{c}$ and $J / \Psi$ mass would certainly be decisive. The best process where to look for a glueball in this mass region is $p \bar{p} \rightarrow \phi \phi$, which is doubly OZI forbidden and should proceed through a purely gluonic state. The cross section for this reaction, if the glueball really appears as an intermediate state in the s-channel, should manifest a resonant behaviour. In the case of the pseudoscalar glueball $P$, the cross section at the peak can be written down as:

$$
\sigma^{P e a k}(p \bar{p} \rightarrow P \rightarrow \phi \phi)=\frac{\pi}{k_{C M}^{2}} \operatorname{Br}(P \rightarrow \phi \phi) \operatorname{Br}(P \rightarrow p \bar{p})
$$

where $k_{C M}$ is the center of mass momentum of the initial hadrons. In order to estimate, albeit roughly, this cross section we can use the results of section 3 which give $\operatorname{Br}(P \rightarrow \phi \phi) \simeq 0.1$ and

$$
\Gamma(P \rightarrow p \bar{p})=\frac{\Gamma\left(\eta_{c} \rightarrow p \bar{p}\right)}{\sin ^{2} \alpha}
$$

Using the experimental value of $\operatorname{Br}\left(\eta_{c} \rightarrow p \bar{p}\right)$ [12 we get

$$
\sigma^{P e a k}(p \bar{p} \rightarrow P \rightarrow \phi \phi) \simeq \frac{1}{\sin ^{2} \alpha}\left(10^{-2} \div 10^{-1}\right) \mu \mathrm{b}
$$

Remarkably, this value increases when $\alpha$ is small and therefore it permits to establish even a small admixture of glueball in the charmonium state.

Taking $\sin ^{2} \alpha \simeq 0.1$, according to the results of the previous section, we obtain the numerical estimate

$$
\sigma^{\text {Peak }}(p \bar{p} \rightarrow P \rightarrow \phi \phi) \simeq\left(10^{-1} \div 1\right) \mu \mathrm{b}
$$

Some experimental data on the $p \bar{p} \rightarrow \phi \phi$ process (although preliminary) are already available from the LEAR experiment at CERN, up to a center of mass energy of $2.4 \mathrm{GeV}$ [16]. The value of the cross section is about $1 \mu \mathrm{b}$ at the highest energy available. It is clearly seen from the data that this cross section decreases with energy. Therefore, according to the our estimate of $\sigma^{\text {Peak }}(p \bar{p} \rightarrow \phi \phi)$, the signal due to the presence of a glueball resonance in the energy region of $\sqrt{s} \simeq 3$ $\mathrm{GeV}$ should be observable above the expected background. Also the cross sections $\sigma(p \bar{p} \rightarrow J / \Psi \rightarrow \phi \phi)$ and $\sigma\left(p \bar{p} \rightarrow \eta_{c} \rightarrow \phi \phi\right)$ are much smaller than the value estimated above.

The observation of this glueball state in the $p \bar{p} \rightarrow \phi \phi$ reaction would therefore be a clear test of the model under investigation.

For what concerns the vector glueball state, it should manifest itself, for instance, in $\Psi^{\prime} \rightarrow \rho \pi X$ decay as a resonance in the $\rho \pi$ effective mass distribution.

Another possibility to observe gluonium is to look for a resonance in the $\phi \phi$ effective mass distribution in the reaction $\pi^{-} p \rightarrow \phi \phi X$.

Notice that the decays which can proceed through the $(c \bar{c})$ component are not much affected by the glueball mixing, since the mixing angle is very small, as we have shown. We expect in these channels effects of the order of $\approx 1 \%$, which, considering 
the theoretical uncertaintes in the charmonium wave functions [17, 18], would be quite difficult to detect even in the next generation experiments.

Finally it can be interesting to note that the mixing angles $\theta$ and $\alpha$ could be experimentally related through the ratio

$$
\frac{\Gamma\left(\eta_{c} \rightarrow \gamma \gamma\right)}{\Gamma\left(J / \Psi \rightarrow e^{+} e^{-}\right)}=1 / 12 \cdot \frac{\left|\Psi_{\eta_{c}}(0)\right|^{2}}{\left|\Psi_{J / \Psi}(0)\right|^{2}}\left(1+1.96 \frac{\alpha_{s}}{\pi}\right) \frac{\cos ^{2} \alpha}{\cos ^{2} \theta}
$$

which gives, assuming $\left|\Psi_{\eta_{c}}(0)\right|=\left|\Psi_{J / \Psi}(0)\right|$,

$$
\frac{\cos ^{2} \alpha}{\cos ^{2} \theta}=0.78 \pm 0.29
$$

in agreement with a larger $\alpha$ than $\theta$. Of course, this value could be much improved in the next future. Incidentally, it must be noted that Ono and Schörbel [19] claim different values for the ratio $\left|\Psi_{\eta_{c}}(0)\right| /\left|\Psi_{J / \Psi}(0)\right|$; however, their model seems to be excluded by the recent data on the charmonium ${ }^{1} P_{1}$ mass [20].

\section{Conclusions}

We can summarize our conclusions by stating that the only available model which could explain the decays of $J / \Psi$ and $\eta_{c}$ forbidden in the usual perturbative QCD treatment of exclusive processes is the one requiring the presence of gluonium states in the proximity of their masses.

In this paper we have shown that such a model is not excluded by the existing data; a tiny gluonic admixture can easily account for the "forbidden" decays and does not significantly change the allowed ones. The model can be easily tested in that one should recover the usual perturbative QCD predictions for the decays of large mass states: in particular the helicity forbidden decays of $\eta_{c}^{\prime}, \Psi^{\prime}, \Upsilon, \Upsilon(2 S)$, etc. should be strongly suppressed.

A direct decisive test of the model can be provided by the experimental observation of a (gluonic) resonance in the $p \bar{p} \rightarrow \phi \phi$ reaction at $\sqrt{s} \simeq 3 \mathrm{GeV}$; we have given an estimate of the corresponding cross section.

\section{References}

[1] S.J. Brodsky and G.P. Lepage, Phys. Rev. D 24, 2848 (1981).

[2] V.L. Chernyak and A.R. Zhitnitsky, Phys. Rep. 112, 173 (1984).

[3] M. Anselmino, F. Caruso and F. Murgia, Phys. Rev. D 42, 3218 (1990).

[4] P.G.O. Freund and Y. Nambu, Phys. Rev. Lett. 34, 1645 (1975); Wei-Shou Hou and A. Soni, Phys. Rev. Lett. 50, 569 (1983). 
[5] M. Anselmino, F. Caruso and S. Forte, Phys. Rev. D 44, 1438 (1991).

[6] M. Anselmino, R. Cancelliere and F. Murgia, Phys. Rev. D 46, 5049 (1992).

[7] S.J. Brodsky, G.P. Lepage and S.F. Tuan, Phys. Rev. Lett. 59, 621 (1987).

[8] M.Anselmino, M. Genovese and E. Predazzi, Phys. Rev. D44, 1597 (1991).

[9] A.S. de Castro and H.F. de Carvalho, J. Phys. G 16, L81 (1990); S. Iwao, Lett. Nuov. Cim. 32, 475 (1981), Quarks 82, Sukhumi Proc. Moscow Editions, p. 228 (1983).

[10] M. Benayoun, V.L. Chernyak and I.R. Zhitnitsky, Nucl. Phys. B348, 327 (1991).

[11] A. R. Zhitnisky et al., Sov. J. Nucl. Phys. 41, 284 (1985).

[12] K. Hikasa et al., Phys. Rev. D45 II (1992).

[13] D. Robson, Nucl. Phys. B130, 328 (1977).

[14] H.S. Chanowitz, Proc. 23th Int. Conf. High Energy Phys., Berkeley, 692 (1986).

[15] R. Cester, private communication.

[16] M. Macrì et al., JETSET coll., Nucl. Phys. A558, 27c (1993).

[17] E. Eichten et al., Phys. Rev. D21, 203 (1980); W. Buchmüller and S.H.H. Tye, Phys. Rev. D24, 132 (1981); A. Martin, Phys. Lett. 100B, 511 (1981); J.L. Richardson, Phys. Lett. 82B, 272 (1979).

[18] A.X. El-Khadra, Nucl. Phys. (Proc Suppl.) B30, 449 (1993); A.X. El-Khadra, Nucl. Phys. (Proc Suppl.) B26, 372 (1992); P.B. Mackenzie, Nucl. Phys. (Proc Suppl.) B26, 369 (1992).

[19] S. Ono and F. Schöberl, Phys. Lett. 118B, 419 (1982).

[20] M. Martini, E760 coll., PANIC Conf., Perugia (1993) 